\title{
Portuguese health care providers' knowledge, attitudes, and acceptability of HIV pre-exposure prophylaxis
}

\author{
Rui Baptista Gonçalves ${ }^{1,2}$, Ana Marreiros², Gonçalo Figueiredo Augusto ${ }^{3}$ \\ ${ }^{1}$ University of Liverpool, School of Medicine, United Kingdom \\ ${ }^{2}$ Departamento de Ciências Biomédicas e Medicina, Universidade do Algarve, Portugal \\ ${ }^{3}$ Global Health and Tropical Medicine, GHTM, Instituto de Higiene e Medicina Tropical, IHMT, Universidade NOVA de Lisboa, \\ UNL, Portugal
}

\begin{abstract}
Introduction: Evidence shows that pre-exposure prophylaxis (PrEP) is highly effective in preventing sexually acquired human immunodeficiency virus (HIV) infection. Whilst its implementation is limited to the US and some countries where demonstration projects have taken place, PrEP has recently been introduced in Portugal. Successful implementation of PrEP requires actively engaging and involving of health care providers (HCP) in Portugal, yet little is known about their knowledge, attitudes, and acceptability towards this novel intervention.

Material and methods: An online survey of convenience-sampled Portuguese HCP was conducted in January 2016, prior to PrEP implementation, to determine their knowledge, attitudes, practices, and acceptability of PrEP.

Results: A total of 96 respondents nationwide took part in the study, of whom the majority were females (60.3\%), specialist physicians/junior doctors (39\%/53\%), working in the field of HIV for 1 to 5 years (42.3\%). Over half (51\%) considered having an average or high knowledge of PrEP, and this was more common amongst infectious diseases specialists. Major concerns regarding PrEP implementation included a rise in sexually transmitted infections and increased antiretroviral resistance. Despite only 31.3\% being asked about PrEP in the future, the majority (75\%) considered that PrEP should be made available in Portugal. The main barrier to PrEP implementation was the lack of knowledge and information to HCP.

Conclusions: Portuguese HCP demonstrated variable degrees of knowledge around PrEP, yet most would support its introduction in the country. Concerns were raised about potential consequences of PrEP roll out as well as implementation challenges that ought to be addressed before PrEP introduction.
\end{abstract}

HIV AIDS Rev 2018; 17, 4: 249-258 DOI: https://doi.org/10.5114/hivar.2018.80256

Key words: prevention, HIV/AIDS, Portugal, PrEP.

\section{Introduction}

In recent years, randomized clinical trials have demonstrated that the use of oral tenofovir disoproxil fumarate (TDF) regimens as pre-exposure prophylaxis (PrEP) in in- dividuals at risk of human immunodeficiency virus (HIV) infection can significantly reduce their risk of acquiring HIV [1-3]. Although results of those trials differ according to targeted populations, PrEP has shown to reduce the risk of HIV acquisition in men who have sex with men (MSM),
Address for correspondence: Dr. Rui Baptista Gonçalves, University of Liverpool, School of Medicine, L69 3GE, Liverpool, United Kingdom,

e-mail: rui.baptistagoncalves@online.liverpool.ac.uk
Article history:

Received: 10.12.2017

Received in revised form: 27.03.2018

Accepted: 02.04.2018

Available online: 20.11.2018
International Journal of HIV-Related Problems

HIV \& AIDS

R e v i e w 
heterosexual persons, and people who inject drugs (PWID). While there have been concerns about the lack of adherence in women participating in two trials, the evidence from studies has consistently shown that adherence is a key predictor to efficacy of PrEP. Therefore, it is important to highlight that PrEP requires strict adherence to the regimen being administered, as this is directly proportional to the level of protection.

In 2015, the World Health Organization (WHO) made a strong recommendation that oral PrEP should be offered to any person at significant risk for HIV as part of combination prevention. This recommendation is a critical step in the global response, and underscores that an effective HIV prevention programme should feature a 'comprehensive package' of interventions, with an aim at reducing the incidence of HIV infection amongst not just key populations but for any individual who is at a higher risk of HIV [4]. Prior to the WHO recommendation and in light of the evidence that consistently showed the effectiveness of PrEP [2], the US Food and Drug Administration (FDA) approved the use of daily tenofovir disoproxil fumarate and emtricitabine (TDF-FTC) as a form of prevention of sexual HIV acquisition [5]. In addition, the European Medicines Agency (EMA) has recommended granting a marketing authorization in the European Union (EU) for TDF-FTC PrEP, in combination with safer sex practices to reduce the risk of sexually-acquired HIV-1 infection in adults at highrisk [6]. Notably, there are 32 countries with current demonstration projects of daily oral PrEP or clinical trials, of which five are in the EU region [7].

The Portuguese Directorate-General for Health estimated that 45,501 [44,470-46,508] people were living with HIV in Portugal in 2016 [8]. Sexual transmission remains the main route of HIV transmission, especially among heterosexual individuals (57.0\% of all new cases in 2016), while MSM accounts for $35.0 \%$ of all new HIV cases in 2016 [8].

A recent study conducted in Portugal reported that $59.3 \%$ of their participants were eligible for PrEP, following the New York State Department of Health AIDS Institute criteria [9]. Other study on acceptability of PrEP among 110 MSM reported that $41.0 \%$ were aware of PrEP. Of these, only $20.0 \%$ had been informed about PrEP through a health care professional (HCP), which can be attributed to both reluctance from HCP in discussing PrEP (as it was not yet available in Portugal) and lack of information to provide to their patients. Furthermore, in that group, 57.0\% reported that they would take PrEP if available. It is also important to highlight that $41.0 \%$ of all participants had been engaged in unprotected sex in past 6 months [10].

Successfully implementing PrEP requires a continuous engagement with HCP in identifying and supporting those that can benefit from offering PrEP. Furthermore, well-trained professionals are a pivotal agent in ensuring correct prescription and monitoring of PrEP users. However, little is known about the readiness, willingness, knowledge, and attitudes towards PrEP in HCP. Most studies on this topic have been conducted in the USA, where PrEP has been available since 2012 [11].
A review from 2012 found only two studies focusing on HCP's awareness and practice of PrEP in the USA [12]. The main concern of professionals was related to the lack of official guidance, which has subsequently been published by both the Centers for Disease Control and Prevention (CDC) and the WHO. Surprisingly, studies conducted after those guidelines were published continue to highlight a need for more information and guidance for HCP, not only in the USA $[11,13,14]$ but also in countries were PrEP is being considered as a part of HIV prevention programming $[15,16]$. Additionally, a qualitative study from 2012 offered further insight into the implementation challenges that HCP faced at the time PrEP was introduced in the USA, including lack of clarity on eligibility criteria and need for additional strategies to be used along with PrEP [17].

Whilst the range of concerns may have changed over time in the USA [18], studies in Canada [15], the UK [16], and Italy [19] demonstrated a relatively similar degree of knowledge, acceptability, and attitudes towards PrEP. Although HCP have variable knowledge of PrEP in those studies, the same key issues are raised in terms of what may impact their willingness to prescribe as well as its acceptability as an effective prevention tool. Concerns over adherence, cost, increase in risk compensation (i.e., the introduction of PrEP may reduce the perceived risk of an individual's sexual behavior and may cause an increase in risky sexual behaviors) and PrEP's effectiveness have all been consistently documented amongst HCP [20]. Nonetheless, acceptability levels have been consistently significant and reported in the $40-80 \%$ range $[15,16,19,21,22]$.

As studies looking at these issues start to emerge in countries considering PrEP implementation, evidence on HCP's perception of PrEP where it is yet to be implemented, such as Portugal, can support public health officials in optimizing planning and service delivery of PrEP. Thus, the aim of this study is to evaluate HCP's knowledge, attitudes, practices, and acceptability regarding the use of oral PrEP in Portugal.

\section{Material and methods}

\section{Study design}

This was an observational cross-sectional study of Portuguese HCP working with people at risk of HIV. It focused on generating evidence on participants' knowledge, attitudes, and practices as well as acceptability towards PrEP.

\section{Sampling and recruitment}

The study used convenience sampling to recruit participants. Eligibility criteria included: being a consenting HCP working with people living with or at risk of HIV infection in Portugal. Exclusion criteria comprised those who were not HCP nor working in supporting people at risk of HIV.

Between December 2015 and January 2016, eligible participants were invited to complete an online survey via email through a range of professional groups and community- 
based organizations. Social media was also employed to distribute the online survey in selected groups of medical professionals. All eleven Portuguese hospitals with a department for infectious diseases (ID) were also approached by email and/ or by telephone as well as a network of general practitioners (GP). Dissemination of information occurred also verbally.

The broad convenience-sampling strategy did not allow estimating the response rate. However, from the current 163 infectious diseases physicians registered with the Portuguese Medical Association in December 2015 [23], 9.8\% participated in this study.

\section{Data collection and ethical considerations}

An online survey was devised for this project, using the SurveyMonkey ${ }^{\oplus}$ platform. The questionnaire was adapted from the study by Desai et al. [16] and based on the literature review. The questionnaire covered knowledge of PrEP, attitudes regarding PrEP, current practice and acceptability of PrEP, and demographics. It included a brief explanation of the study, participants' informed consent to participate, and 23 close-ended questions (multiple choice and Likert scale questions). Before was circulated, the survey was piloted in order to ascertain the feasibility of the questionnaire as well as to identify any logistical and/or issues with its structure and content [24].

The study and the instrument were approved by the Director of the Biomedical Sciences and Medicine Department - University of Algarve, prior to data collection.

\section{Data analysis}

Data from questionnaires were cleaned, exported into an Excel spreadsheet, and analyzed for internal consistency. $\mathrm{IBM}^{\oplus}$ Statistical Package for the Social Sciences (SPSS) version 21.0 for Windows (IBM Corp., Armonk, NY, USA) was used in all data analysis.

Descriptive statistics were conducted for sociodemographic characteristics of study participants. Furthermore, the Categorical Principal Component Analysis (CATPCA) was used to analyze categorical variables. The choice of a multivariable technique is linked to the intention of the study to verify to what extent the instrument can be applied to the Portuguese population. This methodological rationale involves reducing the dimension of the problem using CATPCA. Its purpose lies in the maximum consistency that can be found whilst connecting the items of all considered variables.

Univariate analysis used a $\chi^{2}$ test of proportions or a two-tailed Fisher's exact test where figures were less than five in any group. Missing data were excluded from statistical analyses. Likert scales were used to ask questions about attitudes to clinical management, patient safety, prioritization, and provision of PrEP. Attitudes were ranked from the most negative to the most positive towards PrEP for all respondents, and according to respondents' support or non-support of PrEP introduction in Portugal. Uni- and multivariable logistic regression analysis was used to identify which attitudes were barriers to PrEP availability. Statistical significance was set for $p<0.05$.

\section{Results}

\section{Respondents demographics}

A total of 136 questionnaires were completed, with 40 being excluded from the analysis as they did not meet the eligibility criteria. After data validation, an additional 18 questionnaires were excluded due to missing data. Finally, 78 responses were included in the analysis.

The majority of respondents were women, over the age of 30 years, worked in the field of HIV for 1 to 5 years, and worked in the North or the Lisbon and the Tagus Valley Regions (Table 1). Sixty-one (78.2\%) respondents were doctors, being $38.4 \%$ senior doctors, and $39.7 \%$ junior doctors. Also, $5(6.4 \%)$ respondents were psychologists, $5(6.4 \%)$ were social workers, and 3 (3.8\%) were nurses (Table 1). A range of other professional categories was also recorded as 'other' (5.1\%) including 2 medical students and 2 from other backgrounds.

\section{Knowledge of pre-exposure prophylaxis}

The majority (55.1\%) of respondents considered having a high $(21.8 \%)$ or average $(33.3 \%)$ knowledge of PrEP (Table 2). Nine (11.5\%) participants had never heard of PrEP and 26 (33.3\%) reported having a low level of knowledge of PrEP (Table 2). Analyzing self-reported knowledge of PrEP by profession, doctors (especially senior and junior doctors from infectious diseases) accounted for the majority of those with high level of knowledge of PrEP (Table 2). However, both senior and junior GPs showed low or inexistent knowledge of PrEP. All senior GPs felt they did not have sufficient knowledge of PrEP to have an informed discussion with their patients, while $87.5 \%$ of ID senior doctors felt they did. No significant differences were found regarding variations within the sample for all other demographic categories. Notably, professionals working in ID had greater awareness of the recent WHO guidance on PrEP: only 3 (18.8\%) senior and $2(20.0 \%)$ junior ID doctors were not aware of the document, whereas all GP senior and junior doctors had never read or been informed about it.

A list of PrEP randomised controlled trials (RCTs) was presented to ascertain whether respondents were familiar with them. More respondents were familiar with the Partners PrEP study (28.1\%), followed by the iPrEX study (27.1\%), and the PROUD study (25.0\%). However, 59.4\% of all respondents had never heard of any study. This lack of awareness of any of the seminal clinical trials was higher amongst both senior (90.0\%) and junior (80.0\%) GPs. 
Table 1. Respondents demographics

\begin{tabular}{|c|c|}
\hline Factor & $n(\%)$ \\
\hline Gender & $78(100.0)$ \\
\hline Male & $31(39.7)$ \\
\hline Female & $47(60.3)$ \\
\hline Age & $78(100.0)$ \\
\hline$<29$ & $25(32.1)$ \\
\hline $30-39$ & $38(48.7)$ \\
\hline $40-49$ & $8(10.3)$ \\
\hline $50-59$ & $5(6.4)$ \\
\hline$\geq 60$ & $2(2.6)$ \\
\hline Time working on HIV & $78(100.0)$ \\
\hline$<1$ year & $10(12.8)$ \\
\hline 1 to 5 years & $33(42.3)$ \\
\hline 6 to 15 years & $28(35.9)$ \\
\hline$>15$ years & $7(8.9)$ \\
\hline Region & $78(100.0)$ \\
\hline North & $25(32.1)$ \\
\hline Centre & $17(21.8)$ \\
\hline Greater Lisbon & $25(32.1)$ \\
\hline South & $9(11.5)$ \\
\hline Islands (Madeira/Azores) & $2(2.6)$ \\
\hline Profession & $78(100.0)$ \\
\hline Doctor & $61(78.2)$ \\
\hline Nurse & $3(3.8)$ \\
\hline Psychologist & $5(6.4)$ \\
\hline Social worker & $5(6.4)$ \\
\hline Other & $4(5.1)$ \\
\hline Doctors by medical category & $61(100.0)$ \\
\hline Senior ID & $16(26.2)$ \\
\hline Senior GP & $10(16.4)$ \\
\hline Senior (other) & $4(6.6)$ \\
\hline Junior ID & $10(16.4)$ \\
\hline Junior GP & $5(8.2)$ \\
\hline Junior (other) & $16(26.2)$ \\
\hline
\end{tabular}

\section{Attitudes to pre-exposure prophylaxis}

Despite variable knowledge of PrEP, most respondents (76.9\%) considered that PrEP should be made available in Portugal in light of the current evidence (Table 4). This was particularly the case among nurses (100.0\%), GP senior doctors $(90.0 \%)$, followed by junior ID doctors $(80.0 \%)$, and social workers $(80.0 \%)(p=0.99)$. ID senior doctors represented a lower percentage $(68.8 \%)$ of supporters of the introduction of PrEP in Portugal, only to be overcome by psychologists (60.0\%).
The majority of those supporting PrEP introduction in Portugal felt that it should be targeted to seronegative partners in a serodiscordant (SD) relationship (60.0\%), sex workers $(56.7 \%)$, and all individuals engaging in high-risk behaviors (55.0\%) (Table 3). Lower percentages were found for categories involving one group only, such as PWID (26.7\%) or MSM (21.3\%) (Table 3).

When questioned on specific aspects of clinical usage of PrEP and crossed with overall support of its introduction in Portugal, results showed that those who did not support the introduction of PrEP in Portugal were more inclined towards disagreeing with a broadened access of PrEP (66.7\% vs. $10.0 \%$; $p<0.01)$, considering $\mathrm{PrEP}$ as an inefficient preventative tool (61.1\% vs. $11.7 \%$; $p<0.01$ ) and not feeling comfortable with prescribing PrEP without specific guidelines $(83.3 \%$ vs. $60.0 ; p=0.07)$ (Table 4).

When focusing on access, cost effectiveness of PrEP, and governmental funding, $56.4 \%$ of respondents disagreed that it should be simply offered to those who request it. The majority $(73.1 \%)$ agreed that it should only be provided to those at increased risk of contracting HIV. The prescription regimen that achieved greater concordance was that of making PrEP available but being the cost only partly subsidized by the National Health Service $(50.0 \%)$.

CATPCA was then employed to allow identifying the underlying components of a set of attitude-related items, while maximizing the amount of variance accounted for in those items (by the principal components). According to the Chronbach's a, two broader and statistically consistent dimensions were observed that were then designated by 'indifference' and 'concern' towards potential PrEP users, translated by consistent values of a quantified by 0.854 and 0.730 , respectively.

The multivariable logistic model shows that the odds ratio for HCP supporting PrEP availability in Portugal was lowest when disagreeing with the statement 'PrEP should be widely available based on current evidence' $(p<0.01)$, which suggests that, not surprisingly, this attitude is the greatest barrier to support PrEP introduction in Portugal (Table 5). The second barrier identified in the multivariable logistic model was the concern that patients who take PrEP would be mistakenly labelled as HIV-positive $(p=0.01)$. Other attitudes were important predictors in the univariate model but not in the multivariable model (Table 5). Disagreeing with 'PrEP being an effective preventive tool based on current evidence, and that 'PrEP will have a greater impact than behavioral intervention in preventing HIV infection' were significant at $5 \%$ level in the univariate but not in the multivariable model (Table 5).

With respect to acceptability, participants were asked to rate a number of aspects, which could negatively impact the implementation of PrEP in Portugal (Fig. 1). Answers were grouped in three categories: Agree (covering 
Table 2. Reported knowledge of pre-exposure prophylaxis (PrEP), by professional group

\begin{tabular}{|c|c|c|c|c|c|c|}
\hline \multirow{2}{*}{ Factor } & \multirow{2}{*}{$\begin{array}{l}\text { Overall } \\
n(\%)\end{array}$} & \multicolumn{5}{|c|}{$\begin{array}{c}\text { Professional group } \\
n(\%)\end{array}$} \\
\hline & & $\begin{array}{l}\text { Doctor } \\
n=61\end{array}$ & $\begin{array}{l}\text { Nurse } \\
n=3\end{array}$ & $\begin{array}{c}\text { Psychologist } \\
n=5\end{array}$ & $\begin{array}{c}\text { Social worker } \\
\quad n=5\end{array}$ & $\begin{array}{l}\text { Other } \\
n=4\end{array}$ \\
\hline \multicolumn{7}{|l|}{ Knowledge of PrEP } \\
\hline High & $17(21.8)$ & $13(21.3)$ & $2(66.7)$ & $1(20.0)$ & $0(0.0)$ & $1(25.0)$ \\
\hline Average & $26(33.3)$ & $19(31.1)$ & $0(0.0)$ & $2(40.0)$ & $3(60.0)$ & $2(50.0)$ \\
\hline Low & $26(33.3)$ & $20(32.8)$ & $1(33.3)$ & $2(40.0)$ & $2(40.0)$ & $1(25.0)$ \\
\hline Inexistent/never heard of it & $9(11.5)$ & $9(14.8)$ & $0(0.0)$ & $0(0.0)$ & $0(0.0)$ & $0(0.0)$ \\
\hline \multicolumn{7}{|l|}{ Aware of RCTs } \\
\hline iPrEx & $26(33.3)$ & $22(36.1)$ & $3(100.0)$ & $0(0.0)$ & $0(0.0)$ & $1(25.0)$ \\
\hline FEM-PREP & $12(15.4)$ & $9(14.8)$ & $1(33.3)$ & $0(0.0)$ & $1(20.0)$ & $1(25.0)$ \\
\hline CDC-TDF2 & $11(14.1)$ & $8(13.1)$ & $2(66.7)$ & $0(0.0)$ & $0(0.0)$ & $4(100.0)$ \\
\hline Partners PrEP & $25(32.1)$ & $20(32.8)$ & $2(66.7)$ & $2(40.0)$ & $0(0.0)$ & $1(25.0)$ \\
\hline VOICE & $6(7.7)$ & $4(6.6)$ & $1(33.3)$ & $0(0.0)$ & $0(0.0)$ & $1(25.0)$ \\
\hline PROUD & $24(30.8)$ & $19(31.1)$ & $2(66.7)$ & $2(40.0)$ & $0(0.0)$ & $1(25.0)$ \\
\hline Not aware of any RCT & $42(53.8)$ & $32(52.5)$ & $0(0.0)$ & $3(60.0)$ & $4(80.0)$ & $0(0.0)$ \\
\hline
\end{tabular}

$R C T s$ - randomised controlled trials

Table 3. Distribution of target groups to which pre-exposure prophylaxis should be offered if available, by professional group

\begin{tabular}{l|c|c|c|c|c|c}
\hline & \multirow{7}{*}{$\begin{array}{c}\text { Overall } \\
\text { Target groups }\end{array}$} & \multicolumn{5}{|c}{$\begin{array}{c}\text { Professional group } \\
\text { (\%) }\end{array}$} \\
\cline { 5 - 8 } & $n$ & Doctor & Nurse & Psychologist & Social worker & Other \\
\hline Sexually active MSM & $18(30.0)$ & $13(72.2)$ & $2(11.1)$ & $1(5.6)$ & $0(0.0)$ & $2(11.1)$ \\
\hline MSM with recurrent STIs & $25(41.7)$ & $20(80.0)$ & $1(4.0)$ & $1(4.0)$ & $1(4.0)$ & $2(8.0)$ \\
\hline All MSM & $13(21.7)$ & $11(84.6)$ & $1(7.7)$ & $0(0.0)$ & $0(0.0)$ & $1(7.7)$ \\
\hline All sexually active people with multiple partners & $20(33.3)$ & $17(85.0)$ & $2(10.0)$ & $0(0.0)$ & $0(0.0)$ & $1(5.0)$ \\
\hline Sex workers & $34(56.7)$ & $28(82.3)$ & $2(5.9)$ & $2(5.9)$ & $0(0.0)$ & $2(5.9)$ \\
\hline People who inject drugs & $16(26.7)$ & $12(75.0)$ & $1(6.3)$ & $1(6.3)$ & $0(0.0)$ & $2(12.4)$ \\
\hline $\begin{array}{l}\text { All seronegative people in a monogamous SD } \\
\text { relationship }\end{array}$ & $36(60.0)$ & $30(83.2)$ & $1(2.8)$ & $1(2.8)$ & $2(5.6)$ & $2(5.6)$ \\
\hline Only seronegative MSM in a SD relationship & $2(3.3)$ & $0(0.0)$ & $0(0.0)$ & $0(0.0)$ & $1(50.0)$ & $1(50.0)$ \\
\hline $\begin{array}{l}\text { Only seronegative people in a SD relationship } \\
\text { trying to conceive }\end{array}$ & $5(8.3)$ & $5(100.0)$ & $0(0.0)$ & $0(0.0)$ & $0(0.0)$ & $0(0.0)$ \\
\hline All risk takers & $33(55.0)$ & $25(75.7)$ & $2(6.1)$ & $1(3.0)$ & $2(6.1)$ & $3(9.1)$ \\
\hline Other & $7(11.7)$ & $5(71.4)$ & $2(28.6)$ & $0(0.0)$ & $0(0.0)$ & $0(0.0)$ \\
\hline
\end{tabular}

Note: Respondents were asked to select all that apply

MSM - men who have sex with men, STIs - sexually transmitted infections, SD - serodiscordant

those who agreed or strongly agreed), Undecided, and Disagree (for those disagreeing or strongly disagreeing).

The main concerns regarding the implementation of PrEP in Portugal were lack of information of HCP, followed by lack of specific guidelines to regulate PrEP dispense, and cost for the user (assuming no coverage from the government). The aspect that less concerned respondents was lack of interest in PrEP from key populations (Fig. 1).

\section{Practice of pre-exposure prophylaxis}

A total of $32.1 \%$ of respondents reported to have been ever asked for information and advice on PrEP (Table 6). This proportion was higher among doctors $(72.0 \%)$, particularly ID senior (44.0\%), and junior (12.0\%) doctors. However, the large majority (67.9\%) of respondents had never been asked about PrEP. Among those who were ever asked about PrEP, the majority (52.0\%) were asked 1 to 5 times in 


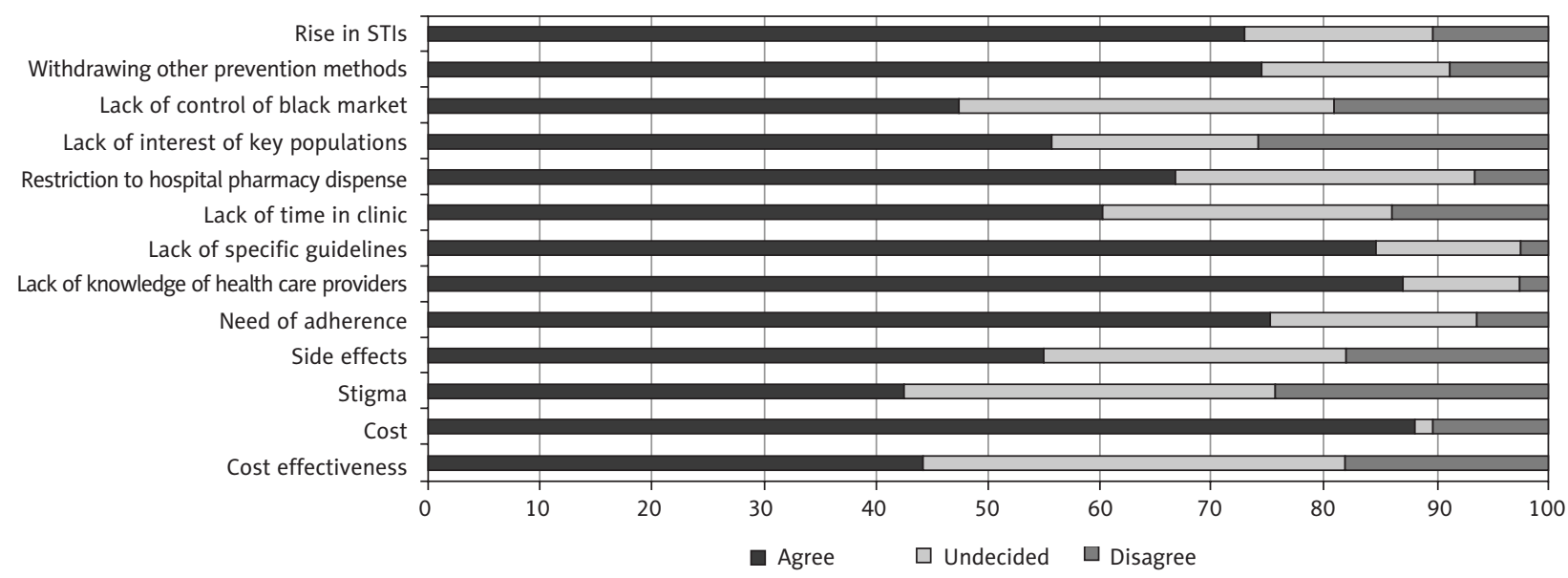

Figure 1. Distribution of Health care providers' agreement with key negative aspects influencing pre-exposure prophylaxis (PrEP) availability

the past year (Table 6), and consisted mostly of ID senior doctors and psychologists.

\section{Discussion}

Portugal has historically and consistently been the country with the highest incidence of HIV infection in Western Europe [25]. In the current Portuguese National HIV Programme, prevention has been identified as one of the key priorities for action [26]. PrEP is an effective tool in HIV prevention, and it is likely that its introduction in Portugal will take place in the near future.

This is the first nationwide survey to assess the knowledge, attitudes, practices, and acceptability of PrEP among HCP in Portugal. When compared to previous studies with HCP [13-15], the study has sampled a broader range of medical specialties and professional categories. It is currently being suggested that PrEP implementation should engage a wider array of HCP rather than solely senior ID doctors $[12,18,22,27]$. The study also surveyed HCP from all regions of the country, thus eliciting the insight of professionals in assorted contexts.

Our study revealed that most respondents were somewhat familiar with PrEP, which was not surprising given that most participants in our study were doctors. However, a degree of unawareness was noted among GPs, which corroborates studies conducted in this group $[13,18]$.

Being familiar with PrEP positively influenced the confidence in discussing PrEP with potential users as well as being aware of the state of the art concerning research on PrEP, which also supports the current evidence [25].

The study found a strong support for the introduction of PrEP in Portugal, particularly if aimed at those at increased risk for HIV infection, and within the cost being partly supported by the user (partial subsidy by the NHS). This finding is consistent with what has been reported from studies conducted in the USA [28], the UK [16], and Italy [19], but approval rate was lower among the Canadian counter- parts [15]. As shown in the British study [16], support for introduction of PrEP was higher amongst senior ID doctors. The study did not report specific reasons for disapproval but highlighted a number of important challenges that may be potential motives. In our survey, PrEP non-supporters were likely to answer that it would have less impact on the epidemic when compared to other behavioral preventative strategies. This group was also skeptical about PrEP effectiveness and, if introduced in Portugal, they would support restricted access to selected potential users. This may reflect existing concerns over whether implementing PrEP will obtain similar results as those reported in clinical trials, in spite of the strong evidence to support its biological efficacy and early guidance, and the endorsement by the CDC and the WHO. The lack of European population data, wide range of adherence rates to PrEP across the different RCTs, and the lack of long-term PrEP efficacy and safety data to demonstrate significant 'real-world' impact may explain such apprehensions.

Concerns over adherence and side effects were less pronounced in our study, when compared with other studies $[16,22,28,29]$. Nevertheless, both PrEP supporters and non-supporters agreed that PrEP may lead to an increase in sexually transmitted infections (STIs) and antiretroviral therapy (ART) resistance, which has also been reported in the literature $[19,28]$. However, data from randomized controlled trials of PrEP suggest that risk of drug resistance is low, occurring in approximately 1 in 1,000 PrEP users in clinical trials [3]. PrEP-selected resistance was observed in the FEM-PrEP study, but this was not frequent [30]. Finally, although some modelling studies suggest a small increase in HIV drug resistance associated with PrEP roll-out, this is not likely to be a major contributor to resistance, if PrEP implementation includes routine monitoring of HIV infection status to limit the possible spread of drug resistance [31].

The concerns expressed by respondents in this study suggest a limited understanding of PrEP RCTs (53.8\% of respondents in our survey were not aware of any RCT). Therefore, PrEP implementation in Portugal should ad- 
Table 4. Variation of agreement and disagreement levels between pre-exposure prophylaxis (PrEP) supporters and non-supporters regarding clinical management, safety, access, and governmental funding for PrEP

\begin{tabular}{|c|c|c|c|c|c|c|}
\hline \multirow[b]{2}{*}{ Statement } & \multicolumn{3}{|c|}{ Agrees or strongly agrees, $n(\%)$} & \multicolumn{3}{|c|}{ Disagrees or strongly disagrees, $n$ (\%) } \\
\hline & $\begin{array}{l}\text { PrEP supporters } \\
\qquad n=60\end{array}$ & $\begin{array}{l}\text { Non-supporters } \\
\text { of PrEP } \\
n=18\end{array}$ & $p$-value & $\begin{array}{l}\text { PrEP } \\
\text { supporters } \\
n=60\end{array}$ & $\begin{array}{l}\text { Non-supporters } \\
\text { of PrEP } \\
n=18\end{array}$ & $p$-value \\
\hline $\begin{array}{l}\text { PrEP should be widely available } \\
\text { based on current evidence }\end{array}$ & $43(71.7)$ & $2(11.1)$ & $<0.01^{*}$ & $6(10.0)$ & $12(66.7)$ & $<0.01^{*}$ \\
\hline $\begin{array}{l}\text { I think PrEP will not be } \\
\text { an effective preventive tool } \\
\text { based on current evidence }\end{array}$ & $7(11.7)$ & $11(61.1)$ & $<0.01^{*}$ & $33(55.0)$ & $1(5.6)$ & $<0.01^{*}$ \\
\hline $\begin{array}{l}\text { I feel comfortable in prescribing } \\
\text { PrEP without specific guidelines }\end{array}$ & $10(16.7)$ & $1(5.6)$ & 0.23 & $36(60.0)$ & $15(83.3)$ & 0.07 \\
\hline $\begin{array}{l}\text { Truvada }^{\circledR} \text { is a safe drug that can } \\
\text { be used as PrEP }\end{array}$ & $33(55.0)$ & $2(11.1)$ & $<0.01^{*}$ & $1(1.7)$ & $6(33.3)$ & $<0.01^{*}$ \\
\hline $\begin{array}{l}\text { The use of PrEP will have little } \\
\text { impact on ART resistance }\end{array}$ & $15(25.0)$ & $1(5.6)$ & 0.07 & $19(31.7)$ & $10(55.6)$ & 0.07 \\
\hline $\begin{array}{l}\text { PrEP will have greater impact } \\
\text { than behavioral intervention on } \\
\text { preventing HIV infection }\end{array}$ & $24(40.0)$ & $2(11.1)$ & $0.02^{*}$ & $23(38.3)$ & $13(72.2)$ & $0.01^{*}$ \\
\hline $\begin{array}{l}\text { The use of PrEP will lead to } \\
\text { an increase in STIs }\end{array}$ & $28(46.7)$ & $12(66.7)$ & 0.14 & $19(31.7)$ & $1(5.6)$ & $0.03^{*}$ \\
\hline $\begin{array}{l}\text { I worry that those who use PrEP } \\
\text { are mistakenly labelled as HIV- } \\
\text { positive }\end{array}$ & $29(48.3)$ & $1(5.6)$ & $<0.01^{*}$ & $18(30.0)$ & $11(61.1)$ & $0.02^{*}$ \\
\hline $\begin{array}{l}\text { I worry that those who use PrEP } \\
\text { will be stigmatized }\end{array}$ & $25(41.7)$ & 3 (16.7) & 0.05 & $16(26.7)$ & $11(61.1)$ & $<0.01^{*}$ \\
\hline $\begin{array}{l}\text { PrEP should be made available } \\
\text { to all those who request it }\end{array}$ & $15(25.0)$ & $0(0.0)$ & $0.02^{*}$ & $29(48.3)$ & $15(83.3)$ & $<0.01^{*}$ \\
\hline $\begin{array}{l}\text { PrEP should be made available } \\
\text { only to those at high risk of HIV } \\
\text { infection }\end{array}$ & $50(83.3)$ & 7 (38.9) & $<0.01^{*}$ & $4(6.7)$ & $4(22.2)$ & 0.06 \\
\hline $\begin{array}{l}\text { PREP should be made available, } \\
\text { but the total cost must be } \\
\text { covered by the user only }\end{array}$ & $11(18.3)$ & $4(22.2)$ & 0.71 & $30(50.0)$ & 7 (38.9) & 0.41 \\
\hline $\begin{array}{l}\text { PREP should be made available } \\
\text { and the cost should be partially } \\
\text { covered by the user }\end{array}$ & $34(56.7)$ & $5(27.8)$ & $0.03^{*}$ & $13(21.7)$ & $9(50.0)$ & $0.02^{*}$ \\
\hline $\begin{array}{l}\text { PREP should be made available } \\
\text { at no cost for the user }\end{array}$ & $18(30.0)$ & $1(5.6)$ & $0.03^{\star}$ & $25(41.7)$ & $14(77.8)$ & $<0.01^{*}$ \\
\hline
\end{tabular}

*Statistically significant at $5 \%$ level

dress the dissemination of PrEP in literature that describes the seminal PrEP trials and presents evidence from the trials in a way that accurately informs HCP.

Respondents considered that PrEP implementation would be most affected by a lack of information and guiding specifically targeting HCP, coupled with costs associated with PrEP. A spectrum of studies has highlighted this need for guidelines and training focused on HCP as PrEP is introduced in settings [14-17, 21]. Notably, in contrast to the Italian study [19], most Portuguese HCP do not feel comfortable in prescribing PrEP without specific guidance.
Regarding cost, our study highlights a level of apprehension towards introducing a potentially expensive preventative intervention that may detract funding from other areas. The international debate on the role of PrEP in the HIV response has also reflected upon this issue $[11,16]$.

Our current study is consistent with the literature in terms of what respondents thought would be groups that were most likely to receive PrEP [19, 28]. Most respondents would offer PrEP to SD partners and people at higher risk for HIV, which may highlight the need to inform providers how to prioritize and identify people at risk that can benefit 
Table 5. Odds ratios of supporting pre-exposure prophylaxis (PrEP) availability when the respondent strongly disagreed with/ disagreed with/undecided about the attitude statement

\begin{tabular}{l|c|c|c|c}
\hline Attitude statement & $\begin{array}{c}\text { Univariable OR } \\
(95 \% \mathrm{Cl})\end{array}$ & $p$-value & $\begin{array}{c}\text { Multivariable OR } \\
(95 \% \mathrm{Cl})\end{array}$ & $p$-value \\
\hline PrEP should be widely available based on current evidence & $0.05(0.01-0.24)$ & $<0.01^{*}$ & $0.03(0.03-0.25)$ & $<0.01^{*}$ \\
\hline PrEP effective preventive tool based on current evidence & $0.09(0.02-0.29)$ & $<0.01^{*}$ & $0.24(0.04-1.53)$ & 0.13 \\
\hline Comfortable prescribing PrEP without specific guidelines $^{*}$ & $0.29(0.04-2.47)$ & 0.26 & $0.66(0.01-51.22)$ & 0.85 \\
\hline Truvada $^{\circledR}$ safe drug for PrEP & $0.41(0.14-1.23)$ & 0.11 & $0.44(0.39-4.91)$ & 0.50 \\
\hline Little impact of PrEP on ART resistance & $0.18(0.20-1.44)$ & 0.11 & $1.93(0.08-46.83)$ & 0.69 \\
\hline $\begin{array}{l}\text { PrEP will have greater impact than behavioral interventions on } \\
\text { preventing HIV infection }\end{array}$ & $0.18(004-0.84)$ & $0.03^{*}$ & $0.38(0.01-4.77)$ & 0.46 \\
\hline PrEP will not lead to an increase in STIs & $2.29(0.76-6.89)$ & 0.14 & $3.95(0.48-32.58)$ & 0.20 \\
\hline Lack of labelling for patients on PrEP & $0.06(0.01-0.50)$ & $<0.01^{*}$ & $0.04(0.01-0.52)$ & $0.01^{*}$ \\
\hline Lack of stigma for patients on PrEP & $0.28(0.07-1.07)$ & 0.06 & $0.46(0.05-4.01)$ & 0.48 \\
\hline
\end{tabular}

*Statistically significant at $5 \%$ level

$\mathrm{Cl}$ - confidence interval, OR - odds ratio, STIs - sexually transmitted infections, ART - antiretroviral therapy

Table 6. Practice of pre-exposure prophylaxis (PrEP), by professional group availability

\begin{tabular}{|c|c|c|c|c|c|c|}
\hline & \multirow{2}{*}{$\begin{array}{c}\text { Overall, } \\
n(\%)\end{array}$} & \multicolumn{5}{|c|}{ Professional group, $n(\%)$} \\
\hline & & $\begin{array}{l}\text { Doctor } \\
n=61\end{array}$ & $\begin{array}{l}\text { Nurse } \\
n=3\end{array}$ & $\begin{array}{l}\text { Psychologist } \\
n=5\end{array}$ & $\begin{array}{l}\text { Social worker } \\
\quad n=5\end{array}$ & $\begin{array}{l}\text { Other } \\
n=4\end{array}$ \\
\hline Ever asked about PrEP by patients & $78(100.0)$ & & & & & \\
\hline Yes & $25(32.1)$ & $18(29.5)$ & $2(66.7)$ & $4(80.0)$ & $0(0.0)$ & $1(25.0)$ \\
\hline No & $53(67.9)$ & $43(70.5)$ & $1(33.3)$ & $1(20.0)$ & $5(100.0)$ & $3(75.0)$ \\
\hline $\begin{array}{l}\text { Number of times asked about PrEP by patients } \\
\text { in the past } 12 \text { months }\end{array}$ & $25(100.0)$ & & & & & \\
\hline 0 & $5(20.0)$ & $5(27.8)$ & $0(0.0)$ & $0(0.0)$ & $0(0.0)$ & $0(0.0)$ \\
\hline $1-5$ & $13(52.0)$ & $10(55.6)$ & $0(0.0)$ & $3(75.0)$ & $0(0.0)$ & $0(0.0)$ \\
\hline$>10$ & $7(28.0)$ & $3(16.7)$ & $2(100.0)$ & $1(25.0)$ & $0(0.0)$ & $1(100.0)$ \\
\hline
\end{tabular}

from PrEP. It also reflects the effect of current practice, given the fact that a third of study sample had already been asked for information on PrEP (mostly by SD partners and MSM). This further underscore the need to develop specific guidance for Portuguese HCP.

The only previous survey looking at PrEP acceptability in Portugal aimed at MSM revealed that more than half of those surveyed were willing to use PrEP, highlighting a level of interest from the users' perspective [12]. Furthermore, it showed that HCP were not the main source of information on PrEP for that sample, which further enhances the need to supply HCP with knowledge and support on the range of issues that encompass PrEP and its implementation. Given the latest WHO recommendation on comprehensive packages for HIV prevention, PrEP is at the core of potentially offering a solution to curtail the epidemic [32]. Our study presents a pragmatic starting point to assess acceptability towards PrEP on a group of key stakeholders in its eventual implementation.

There were some notable limitations in our study. Firstly, there is some weakness associated with the number of respon- dents and the use of convenience sampling, which hindered the correct estimation of the response rate. The study would have benefited from a more in-depth analysis of the demographic variables, with particular emphasis on geographic referencing analyzed in conjunction with the remaining statistical outputs. Details on marital status, religion, HIV status, and sexual orientation of the sample would also have helped to understand whether these influenced the results. Unlike other studies [19], time constraints did not permit a longer period to survey completion and possible qualitative interviews to inform our survey.

Participation was voluntary, and therefore not all eligible candidates may have been willing to complete the survey, albeit having accessed and/or being aware of it; there may be systematic bias between the types of participants who chose to respond and those who did not. An online survey might carry some disadvantages, namely issues of Internet access or potential lack of cooperation for excessive demand to participate in similar studies. These were mitigated, to some extent, by following-up on all of those who were approached to assist with recruitment. 
Other limitations relate to the type of instrument used. Likert scales have been known to encompass a number of drawbacks [33], namely respondents' avoidance to use extreme response categories (central tendency bias), agreement with statements as presented (acquiescence bias), or trying to portray themselves in a more favorable light (social desirability bias). In this study, acquiescence bias may be dissipated by the use of a balanced scale. Similarly, previous studies have shown that online surveys have the potential to limit social desirability bias [19, 34]. Multi-scale items validity check using test-retest was not conducted, but inter-reliability analysis of Chronbach's a confirmed internal consistency. Given that the study was conducted prior to the implementation of PrEP in the country, it would be beneficial to appraise whether these attitudes, knowledge and acceptability have changed with the introduction of PrEP across the country.

\section{Conclusions}

The implementation of PrEP in Portugal requires active engagement with HCP and other stakeholders in health service provision. As HCP are instrumental in implementing a new intervention; it is critical to have a nuanced understanding of their knowledge and acceptability of PrEP. Our study highlighted the wider acceptability of Portuguese HCP towards PrEP in a moment its introduction in the country was being discussed but not yet in course.

In addition, our survey demonstrated that Portuguese HCP were willing to support PrEP implementation efforts, but a range of issues related to specific guideline development, PrEP education programs to HCP, and public financing to potential users must be addressed. Our study also showed that levels of knowledge varied between professional groups, which further accentuates the need for guidance and training on PrEP to HCP. Moreover, specific issues such as lack of information for HCP and cost were identified as potential implementation challenges, which must be taken into account if and when PrEP is implemented in Portugal.

Finally, the results have contributed to our understanding of HCP baseline knowledge, attitudes, practice, and acceptability towards PrEP, and help to fill a knowledge gap in this issue. It also postulated important findings with regards to how these were organized, eliciting diverse degrees of awareness with regard to distinct levels of support. Further research should help to understand concerns related to PrEP raised by HCP in this study.

Bearing in mind the epidemiological trends of HIV infection in Portugal, engaging with novel preventative and cost-effective approaches such as PrEP may carry sufficient public health impact to curb the epidemic.

\section{Conflict of interest}

The authors declare no potential conflicts of interest with respect to the research, authorship, and/or publication of this article.

\section{References}

1. Cohen MS, Chen YQ, McCauley M, et al. Prevention of HIV-1 infection with early antiretroviral therapy. N Engl J Med 2011; 365: 493-505.

2. Okwundu CI, Uthman OA, Okoromah CA. Antiretroviral pre-exposure prophylaxis (PrEP) for preventing HIV in high-risk individuals. Cochrane Database Syst Rev 2012; 7: CD007189.

3. WHO. Guidance on Pre-Exposure oral prophylaxis (PrEP) for serodiscordant couples, men and transgender women who has sex with men at high risk of HIV: Recommendations for use in the context of demonstration projects. WHO, Geneva 2012.

4. WHO. WHO Expands Recommendation on Oral Pre-Exposure Prophylaxis of HIV Infection (PrEP). Available at: http://apps.who. int/iris/bitstream/10665/197906/1/WHO_HIV_2015.48_eng.pdf (Accessed: 15 January 2016).

5. Elion R, Coleman M. The preexposure prophylaxis revolution: from clinical trials to routine practice: implementation view from the USA. Curr Opin HIV AIDS 2016; 11: 67-73.

6. European Medicines Agency. First medicine for HIV pre-exposure prophylaxis recommended for approval in the EU. 22 July 2016. Available at: http://www.ema.europa.eu/ema/index.jsp?curl=pages/news_and_events/news/2016/07/news_detail_002578.jsp\&mid =WC0b01ac058004d5c1 (Accessed: 4 June 2017).

7. AVAC. Ongoing and Planned PrEP Open Label, Demonstration and Implementation Projects, as of May 2017. Available at: http:// www.avac.org/sites/default/files/resourcefiles/ongoing_planned_ oral_PrEP_studies_Dec2016.pdf (Accessed: 4 June 2017).

8. DGS - Portuguese Directorate-General for Health. Portugal: Infeção por VIH, SIDA e Tuberculose em números. DGS, Lisbon 2015.

9. Barros H, Rocha LM, Meireles P, et al. Eligibility for PrEP among members of a HIV-negative MSM Portuguese Cohort. New Orleans, LA: oral communication at APHA $142^{\text {nd }}$ Annual Meeting \& Expo, 15-19 November 2014.

10. Rocha LM, Campos MJ, Brito J, et al. Acceptability of PrEP among HIV negative Portuguese men who have sex with men that attended 2014 Lisbon pride fair. J Int AIDS Soc 2014; 17 (4 Suppl 3): 19734.

11. White JM, Mimiaga MJ, Krakower DS, Mayer KH. Evolution of Massachusetts physician attitudes, knowledge, and experience regarding the use of antiretrovirals for HIV prevention. AIDS Patient Care STDS 2012; 26: 395-405.

12. Krakower D, Mayer KH. Engaging healthcare providers to implement HIV pre-exposure prophylaxis. Curr Opin HIV AIDS 2012; 7: 593-599.

13. Conniff J, Evensen A. Preexposure Prophylaxis (PrEP) for HIV Prevention: The Primary Care Perspective. Am Board Fam Med 2016; 29: 143-151.

14. Seidman D, Carlson K, Weber S, et al. United States family planning providers' knowledge of and attitudes towards preexposure prophylaxis for HIV prevention: a national survey. Contraception 2016; 93: 463-469.

15. Sharma M, Wilton J, Senn H, et al. Preparing for PrEP: perceptions and readiness of Canadian physicians for the implementation of HIV pre-exposure prophylaxis. PLoS One 2014; 9: e105283.

16. Desai M, Gafos M, Dolling D, et al.; PROUD study. Healthcare providers' knowledge of, attitudes to and practice of pre-exposure prophylaxis for HIV infection. HIV Med 2016; 17: 133-142.

17. Arnold EA, Hazelton P, Lane T, et al. A qualitative study of provider thoughts on implementing pre-exposure prophylaxis (PrEP) in clinical settings to prevent HIV infection. PLoS One 2012; 7: e40603.

18. Krakower DS, Mayer KH. The role of healthcare providers in the roll out of preexposure prophylaxis. Curr Opin HIV AIDS 2016; 11: 41-48.

19. Puro V, Palummieri A, De Carli G, et al. Attitude towards antiretroviral Pre-Exposure Prophylaxis (PrEP) prescription among HIV specialists. BMC Infect Dis 2013; 13: 217. 
20. Baeten J, McCormack S. Welcome to the preexposure prophylaxis revolution. Curr Opin HIV AIDS 2016; 11: 1-2.

21. Tripathi A, Ogbuanu C, Monger M, et al. Preexposure prophylaxis for HIV infection: healthcare providers' knowledge, perception, and willingness to adopt future implementation in the southern US. South Med J 2012; 105: 199-206.

22. Cowan E, Calderon Y, Chacón K, et al. Knowledge of and experience with HIV pre-exposure prophylaxis (PrEP) among New York state healthcare providers - THPE190 - Poster Exhibition at the 20th International AIDS Conference - Melbourne, 20-25 July 2014.

23. Ordem dos Médicos - Portuguese Medical Association. Distribuição por Especialidades, Idade e Sexo [Distribution by Specialization, Age and Sex]. Lisbon: Conselho Nacional Executivo da Ordem dos Médicos, 2015.

24. Van Teijlingen E, Hundley V. The importance of pilot studies. Nurs Stand 2002; 16: 33-36.

25. UNAIDS. Portugal: Country Profile. Available at: http://www.unaids. org/en/regionscountries/countries/portugal/ (Accessed: 13 January 2016).

26. DGS - Portuguese Directorate-General for Health. Programa Nacional de Prevenção e Controlo da Infeção VIH/SIDA 2012-2016 (National Programme on HIV/AIDS Prevention and Control 2012-2016). Available at: http://pnvihsida.dgs.pt/programa-nacional111111111.aspx (Accessed: 15 January 2016).

27. Hoffman S, Guidry JA, Collier KL, et al. A Clinical Home for Preexposure Prophylaxis: Diverse Health Care Providers' Perspectives on the "Purview Paradox". J Int Assoc Provid AIDS Care 2016; 15: 59-65.

28. Tellalian D, Maznavi K, Bredeek UF, Hardy WD. Pre-exposure prophylaxis (PrEP) for HIV infection: results of a survey of HIV healthcare providers evaluating their knowledge, attitudes, and prescribing practices. AIDS Patient Care STDS 2013; 27: 553-559.

29. Liegler T, Abdel-Mohsen M, Bentley LG, et al. HIV-1 drug resistance in the iPrEx preexposure prophylaxis trial. J Infect Dis 2014; 210: 1217-1227.

30. Liegler T, Abdel-Mohsen M, Defechereuz P, et al. Drug resistance among HIV-1 seroconverters in the FEM-PrEP study. Secondary Drug resistance among HIV-1 seroconverters in the FEM-PrEP study. 2012. Available at: http://www.informedhorizons.com/resistance2012/pdf/Presentations/Liegler.pdf (Accessed: 15 January 2016).

31. Abbas UL, Hood G, Wetzel AW, Mellors JW. Factors influencing the emergence and spread of HIV drug resistance arising from rollout of antiretroviral pre-exposure prophylaxis (PrEP). PLoS One 2011; 6: e18165.

32. Mugo NR, Ngure K, Kiragu M, et al. The preexposure prophylaxis revolution; from clinical trials to programmatic implementation. Curr Opin HIV AIDS 2016; 11: 80-86.

33. Jamieson S. Likert scales: how to (ab) use them. Med Educ 2004; 38: 1217-1218.

34. Krakower DS, Mimiaga MJ, Rosenberger JG, et al. Limited Awareness and Low Immediate Uptake of Pre-Exposure Prophylaxis among Men Who Have Sex with Men Using an Internet Social Networking Site. PLoS One 2012; 7: e33119. 